Kyoto University,

Graduate School of Economics

Research Project Center Discussion Paper Series

Is the Long-run Equilibrium Wage-led or Profit-led?

A Kaleckian Approach

Hiroaki Sasaki

Discussion Paper No. E-11-002

Research Project Center

Graduate School of Economics

Kyoto University

Yoshida-Hommachi, Sakyo-ku

Kyoto City, 606-8501, Japan

April 2011 


\title{
Is the Long-run Equilibrium Wage-led or Profit-led? A Kaleckian Approach
}

\author{
Hiroaki SASAKI \\ Graduate School of Economics, Kyoto University, Yoshida-Honmachi, Sakyo-ku, Kyoto \\ 606-8501, Japan; e-mail: sasaki@econ.kyoto-u.ac.jp
}

\begin{abstract}
This paper presents a Kaleckian growth model in which (i) the rate of capacity utilization, the profit share, and the rate of employment are adjusted in the medium run, and (ii) the normal rate of capacity utilization and the expected rate of growth are adjusted in the long run. Both the Kalecki type and the Marglin-Bhaduri type investment functions are introduced. Using the model, we examine which regime is obtained in the long-run equilibrium, the wage-led regime or the profit-led regime.
\end{abstract}

Keywords: Kaleckian model; long-run equilibrium; wage-led; profit-led JEL Classification: E12; E24; O41 


\section{Introduction}

How do changes in wages affect the performance of economy? On the one hand, wages are costs: an increase in wages lowers the profit of the firms, and thus, decreases the investment of firms, which consequently, has a negative effect on production and employment. On the other hand, wages are income: an increase in wages stimulates consumption demand, and thus, induces the investment of firms, which consequently, has a positive effect on production and employment. The effect of an increase in wages on the performance of the economy differs depending on which effect dominates.

In post-Keynesian models, especially in Kaleckian models, these differences are explained by the differences in regimes. ${ }^{1}$ Here, the regime is a characteristic of the equilibrium. In particular, the regime is defined by the relationship between the profit share (i.e., the ratio of profit income to nominal national income) and the rate of capacity utilization and by the relationship between the profit share and the rate of capital accumulation. If an increase in the profit share decreases (increases) the rate of capacity utilization and the rate of capital accumulation, then we call it the wage-led (profit-led) regime. In the wage-led regime, an increase in the wage share has a positive effect on production and employment, while in the profit-led regime, it has a negative effect on production and employment.

Many early studies investigate which regime is obtained under what circumstances. Which economic policy is effective depends on a kind of regime. Therefore, regime analysis is important.

Almost all existing studies consider the regime in the short-run or the medium-run equilibrium. Here, the short run or the medium run relates to the period in which the variables of the model such as the rate of capacity utilization, the rate of accumulation, and the profit share are adjusted. For this reason, the objective of these existing studies concerns the effectiveness of an economic policy in the short run or the medium run.

However, the long-run regime has been seldom discussed. Accordingly, the existing theory cannot address the effectiveness of an economic policy in the long run. To consider a long-run situation, we need a long-run theory. Here, we define the long run as the period in which the normal rate of capacity utilization and the expected rate of growth are adjusted. These two variables are treated as constants in the short run and the medium run. The normal rate of capacity utilization is the rate of capacity utilization that firms determine through convention, production experience, and strategic considerations. In the short run and the medium run of the Kaleckian model, the actual rate of capacity utilization diverges from the normal rate of capacity utilization even in the equilibrium. However, in the long run, such

\footnotetext{
${ }^{1}$ For the framework of the Kaleckian model, see Rowthorn (1981) and Lavoie (1992). For various regimes in the Kaleckian model, see Marglin and Bhaduri (1990), Bhaduri and Marglin (1990), and Blecker (2002).
} 
divergence will be adjusted. In addition, it is reasonable to consider that the expectation is adjusted.

Some early studies investigate such a long-run adjustment in the Kaleckian model. These studies sought to challenge the criticism of Marxists and Sraffians. These studies claim that the Kaleckian model is a short-run/medium-run model and not a long-run model (Auerbach and Skott, 1988; Duménil and Lévy, 1999; Park, 1997). Critics assert that in the long run, the divergence between the actual rate of capacity utilization and the normal rate of capacity utilization should vanish: all variables are fully adjusted; consequently, we have the return of classical economics.

In contrast to this criticism, Lavoie (1995, 2003), Dutt (1997), and Cassetti (2006) introduce the long-run adjustment process of the normal rate of capacity utilization in the Kaleckian model. In such extended models, Kaleckian results are obtained even in the long run. For example, "the paradox of thrift" and "the paradox of cost" hold even in the long run. Here, the paradox of thrift means that an increase in the capitalists' savings rate lowers the rate of capital accumulation. The paradox of cost means that an increase in the real wage leads to an increase in the realized rate of profit.

However, even such extended models do not satisfactorily examine which regime holds in the long run.

It is well known that in the short-run and the medium-run analyses, the use of Marglin and Bhaduri's (1990) investment function (MB type investment function) produces various regimes depending on the conditions. In the MB type investment function, the profit share and the rate of capacity utilization determine investment. We apply this MB type investment function to the long-run analysis, and investigate which regime is obtained in the long-run equilibrium. For comparison, we also use the conventional Kalecki type investment function, in which the rate of profit and the rate of capacity utilization determine investment. It is known that in the short-run and the medium-run equilibria with the Kalecki type investment function, both the stagnationist regime and the wage-led growth regime hold. However, the long-run equilibrium with the Kalecki type investment function, too, has not been discussed in detail. ${ }^{2}$ To sum up, this paper contributes to the long-run analysis.

Another contribution of this paper concerns the analysis of the rate of employment. The existing Kaleckian models do not satisfactorily investigate the rate of employment. These models consider that labor supply is unlimited and that the firms employ as many workers as they desire at the given wages. However, it is reasonable to think that labor supply is constrained in the long run. In this case, the equalization of labor demand growth and labor supply growth is an accidental occurrence. Thus, if the labor supply growth steadily

\footnotetext{
${ }^{2}$ Cassetti (2006) uses the Kalecki type investment function in the long-run analysis.
} 
exceeds the labor demand growth, then the rate of employment will be zero; however, this is unrealistic. Therefore, to determine the long-run rate of employment, we need a model in which the economically meaningful employment rate is endogenously determined.

Some papers present Kaleckian models in which the rate of employment is endogenously determined (Dutt, 1992; Lima, 2004; Stockhammer, 2004; Sasaki, 2010). However, from our viewpoint, these models are only concerned with the medium-run analysis, that is, they do not consider the long-run analysis. In our model, the rate of employment is endogenously determined in both the medium run and the long run. From this, we can see the factor that affects the rate of employment. For this purpose, we introduce into the Kaleckian model, a specification relating the rate of employment with the growth rate of labor productivity, as in Bhaduri (2006), Dutt (2006), and Flaschel and Skott (2006).

The remainder of the paper is organized as follows. Section 2 conducts a mediumrun analysis. In the medium run, the rate of capacity utilization, the profit share, and the employment rate are adjusted and endogenously determined. Section 3 conducts a longrun analysis. In the long-run, the normal rate of capacity utilization and the expected rate of growth are adjusted and endogenously determined. Moreover, this section investigates which regime is obtained in the long-run equilibrium. Section 4 concludes the paper.

\section{Medium-run equilibrium}

Consider an economy with workers and capitalists. Suppose that the workers consume all their wages and the capitalists save a fraction $s$ of their profit: only the capitalists save. Then, the ratio of real savings $S$ to the capital stock $K$, that is, $g_{s}=S / K$ leads to

$$
g_{s}=s r, \quad 0<s \leq 1,
$$

where $r$ denotes the rate of profit.

Suppose that the firms operate with the following fixed coefficient production function:

$$
Y=\min \{a L,(u / k) K\}
$$

where $Y$ denotes the real output; $L$, employment; and $a=Y / L$, the level of labor productivity. The rate of capacity utilization $u$ is defined as $u=Y / Y^{*}$, where $Y^{*}$ denotes the potential output. The coefficient $k=K / Y^{*}$ denotes the ratio of the capital stock to the potential output, which is assumed to be constant. This assumption implies that both $K$ and $Y^{*}$ grow at the same rate. Moreover, when the rate of capacity utilization is constant, the growth rate of capital stock and that of the actual output will be the same. Accordingly, the actual output and the potential output grow at the same rate in the equilibrium where the rate of capacity 
utilization is constant. To simplify the analysis, in what follows, we assume that $k=1$. From this, we have $r=m u$, where $m$ denotes the profit share.

Let us specify the firms' planned investment. As stated above, we introduce two kinds of investment function.

For the Kalecki type investment function, we specify the ratio of the real investment $I$ to the capital stock, $g_{d}=I / K$ as follows:

Kalecki type : $g_{d}=\gamma+\delta r+\varepsilon\left(u-u_{n}\right), \quad \gamma \in(0,1), \delta \in(0,1), \varepsilon \in(0,1), \gamma>\varepsilon u_{n}$,

where $\gamma$ denotes a constant term capturing the expected rate of growth, and $u_{n}$, the normal rate of capacity utilization. Note that the investment function is increasing in both the rate of profit and the gap between the actual rate of capacity utilization and the normal rate of capacity utilization. The specification that $u-u_{n}$ is an explanatory variable is adopted by Amadeo (1986) and Lavoie (2006). If the actual rate of capacity utilization is equal to the normal rate of capacity utilization $\left(u=u_{n}\right)$, the firms expand plants at the rate of $g_{d}=\gamma+\delta r$. If, however, the actual rate of capacity utilization is less than the normal rate of capacity utilization $\left(u<u_{n}\right)$, the firms consider themselves as facing excess capacity, and they decrease the rate of capital accumulation to a level lower than in the case of $u=u_{n}$. If, on the other hand, the actual rate of capacity utilization exceeds the normal rate of capacity utilization, the firms increase the rate of capital accumulation to a level higher than in the case of $u=u_{n}$. Finally, the constraint $\gamma>\varepsilon u_{n}$ means that $g_{d}>0$ even if both $u$ and $r$ are zero.

For the MB type investment function, we specify $g_{d}$ as follows:

$$
\text { MB type : } g_{d}=\gamma+\delta m+\varepsilon\left(u-u_{n}\right), \quad \gamma \in(0,1), \delta \in(0,1), \varepsilon \in(0,1), \gamma>\varepsilon u_{n} \text {. }
$$

Marglin and Bhaduri (1990) state that it is not the rate of profit but the profit share that should be an explanatory variable of the investment function. In this case, it is well known that we obtain different regimes in the equilibrium depending on which effect dominates, the effect of the profit share or the effect of the rate of capacity utilization.

Now that we present the basic framework of the model, we turn to the derivation of the dynamical equations for the medium-run analysis.

To begin with, we specify the adjustment of the rate of capacity utilization. In the goods market, excess demand leads to a rise in the rate of capacity utilization while excess supply leads to a decline in the rate of capacity utilization.

$$
\dot{u}=\alpha\left(g_{d}-g_{s}\right), \quad \alpha>0,
$$

where $\alpha$ denotes the speed of adjustment of the goods market. 
From the definition of the profit share, we have $m=1-(w L / p Y)$, where $w$ denotes the money wage, and $p$, the price of goods. Differentiating the profit share with respect to time, we obtain the following relationship

$$
\frac{\dot{m}}{1-m}=\frac{\dot{p}}{p}-\frac{\dot{w}}{w}+\frac{\dot{a}}{a} .
$$

To know the dynamics of the profit share, we must specify the dynamics of $p, w$, and $a$ of equation (6).

We specify the dynamics of the money wage and price by using the theory of conflictingclaims inflation. ${ }^{3}$ First, suppose that the growth rate of the money wage that the workers manage to negotiate depends on the discrepancy between their target profit share and the actual profit share. Second, suppose that the firms set their price to close the gap between their target profit share and the actual profit share. From these considerations, the dynamics of the money wage and price can be described, respectively, as follows:

$$
\begin{aligned}
& \frac{\dot{w}}{w}=(1-\theta)\left(m-m_{w}\right), \quad m_{w} \in(0,1), \\
& \frac{\dot{p}}{p}=\theta\left(m_{f}-m\right), \quad \theta \in(0,1), m_{f} \in(0,1),
\end{aligned}
$$

where $\theta$ is a positive parameter, which captures the bargaining power of the firms, and $1-\theta$ is the bargaining power of the workers. The parameter $m_{w}$ denotes the target profit share set by the workers, and $m_{f}$, the target profit share set by the firms. ${ }^{4}$ In the following analysis, we assume that $m_{f}>m_{w}$. The firms attempt to set their targets as high as possible whereas the workers attempt to set their targets as low as possible. Therefore, the assumption that $m_{f}>m_{w}$ is reasonable.

We now turn to the specification of the endogenous technological change. As stated above, we assume that the growth rate of labor productivity $g_{a}$ depends positively on the rate of employment $e$.

$$
g_{a}=\lambda e^{\beta}, \quad \lambda>0, \beta>0,
$$

\footnotetext{
${ }^{3}$ The theory of conflicting-claims inflation was originally developed by Rowthorn (1977). For the Kaleckian models with conflicting-claims inflation, see also Dutt (1987), Lavoie (1992), and Cassetti (2002, 2003, 2006).

${ }^{4}$ In our model, $m_{w}$ and $m_{f}$ are given exogenously. Naturally, we can endogenize them. For example, it is possible that $m_{w}$ is a decreasing function of $g_{a}$ whereas $m_{f}$ is an increasing function of $g_{a}$. The former specification captures the effect wherein the labor unions attempt to reflect the fruit of an increase in labor productivity in higher wages, whereas the latter specification captures the effect wherein the firms attempt to reflect the fruit of an increase in labor productivity in higher profit. As will be shown by equation (9), $g_{a}$ is an increasing function of $e$, and hence, $m_{w}$ is a decreasing function of $e$ while $m_{f}$ is an increasing function of $e$. With these modifications, the positive effect of $m_{f}^{\prime}(e)>0$ has an effect of stabilizing the medium-run equilibrium while the negative effect of $m_{w}^{\prime}(e)<0$ has a destabilizing effect on the medium-run equilibrium.
} 
where $g_{a}=\dot{a} / a$. The rate of employment is defined as $e=L / N$, where $N$ denotes the exogenous labor supply. The coefficient $\lambda$ denotes a positive constant, and $\beta$, the elasticity of the growth rate of labor productivity with respect to the rate of employment. A rise in the employment rate has an upward pressure on wages. The firms facing this upward pressure tend to adopt labor-saving techniques. ${ }^{5}$ We use the above specification to explicitly solve for the rate of employment. Note, however, that our results do not depend on this specification as long as $g_{a}$ is an increasing function of $e .^{6}$

Let us derive an equation of motion for the rate of employment. From equation (2), the rate of employment is given by $e=u K /(a N)$, from which the rate of change in $e$ leads to

$$
\frac{\dot{e}}{e}=\frac{\dot{u}}{u}+g_{d}-g_{a}-n,
$$

where $n$ denotes the growth rate of $N$ and is given exogenously.

\subsection{Medium-run with Kalecki type investment function}

In conventional Kaleckian models, the variables $u$ and $m$ are adjusted in the short run or medium run. In our medium-run model, in contrast, the three variables $u, m$, and $e$ are adjusted. Substituting equations (1), (3), (7), (8), and (9) in equations (5), (6), and (10), we obtain the following dynamical system:

$$
\begin{aligned}
\dot{u} & =\alpha\left[\gamma+\delta m u+\varepsilon\left(u-u_{n}\right)-s m u\right], \\
\dot{m} & =-(1-m)\left[m-\Gamma-g_{a}(e)\right], \quad \text { where } \Gamma \equiv \theta m_{f}+(1-\theta) m_{w}, \\
\dot{e} & =e\left\{\frac{\alpha\left[\gamma+\delta m u+\varepsilon\left(u-u_{n}\right)-s m u\right]}{u}+\gamma+\delta m u+\varepsilon\left(u-u_{n}\right)-g_{a}(e)-n\right\} .
\end{aligned}
$$

Here, we assume that $\Gamma>n$. This assumption means that the weighted average of the two groups' target profit shares is larger than the growth rate of exogenous labor supply. Given that the size of $n$ is about 10 percent at most, this assumption is plausible. Because $\Gamma=\theta m_{f}+(1-\theta) m_{w}$, an increase in $m_{f}$ or $m_{w}$ corresponds to an increase in $\Gamma$. Moreover, because $m_{f}>m_{w}$, an increase in $\theta$ corresponds to an increase in $\Gamma$. Furthermore, we assume that $s>\delta$. This means that the capitalists' savings rate is larger than the sensitivity of investment with respect to the rate of profit, which is called the Keynesian stability condition.

The medium-run equilibrium is a situation wherein $\dot{u}=\dot{m}=\dot{e}=0$; using this, we obtain the following quadratic equation for the profit share:

$$
(s-\delta) m^{2}-\Theta m+\varepsilon(\Gamma-n)=0, \quad \text { where } \Theta \equiv s\left(\gamma-\varepsilon u_{n}\right)+(s-\delta)(\Gamma-n)+\varepsilon>0 .
$$

\footnotetext{
${ }^{5}$ For a Kaleckian model that considers such an effect, see also Sasaki (2010).

${ }^{6}$ Indeed, the stabilization analysis and the comparative static analysis that will be shown later are conducted with the general form of $g_{a}=g_{a}(e)$.
} 
From equation (14), we obtain two real and distinct roots. However, the larger root corresponds to the medium-run equilibrium value. ${ }^{7}$

$$
m^{*}=\frac{\Theta+\sqrt{\Theta^{2}-4 \varepsilon(s-\delta)(\Gamma-n)}}{2(s-\delta)} .
$$

Hereafter, the medium-run equilibrium values are denoted with “*." Using equation (15), we obtain the rates of capacity utilization and employment in the medium-run equilibrium:

$$
\begin{aligned}
u^{*} & =\frac{\gamma-\varepsilon u_{n}}{(s-\delta) m^{*}-\varepsilon}, \\
e^{*} & =\left(\frac{m^{*}-\Gamma}{\lambda}\right)^{\frac{1}{\beta}} .
\end{aligned}
$$

For the rate of capacity utilization to be positive, we need $(s-\delta) m^{*}-\varepsilon>0$, which can be rewritten as $m^{*}>\varepsilon /(s-\delta)$. In addition, we assume that $m^{*}, u^{*}$, and $e^{*}$ are larger than zero and less than unity.

To analyze the local stability of the medium-run equilibrium, we linearize equations (11), (12), and (13) around the equilibrium, and investigate the corresponding Jacobian matrix. From this procedure, we obtain the following proposition:

Proposition 1. Suppose that the coefficients of the investment function with respect to the rate of profit and the rate of capacity utilization are not extremely large. Then, the mediumrun equilibrium is locally stable.

Proof. See Appendix C.

In the following analysis, we assume that the coefficients of the investment function are not extremely large.

\subsection{Medium-run analysis with MB type investment function}

Following the same procedure as in the case with the Kalecki type investment function, we obtain the following dynamical system:

$$
\begin{aligned}
\dot{u} & =\alpha\left[\gamma+\delta m+\varepsilon\left(u-u_{n}\right)-s m u\right], \\
\dot{m} & =-(1-m)\left[m-\Gamma-g_{a}(e)\right], \quad \text { where } \Gamma \equiv \theta m_{f}+(1-\theta) m_{w}, \\
\dot{e} & =e\left\{\frac{\alpha\left[\gamma+\delta m+\varepsilon\left(u-u_{n}\right)-s m u\right]}{u}+\gamma+\delta m+\varepsilon\left(u-u_{n}\right)-g_{a}(e)-n\right\} .
\end{aligned}
$$

\footnotetext{
${ }^{7}$ See Appendix A.
} 
The medium-run equilibrium is a situation wherein $\dot{u}=\dot{m}=\dot{e}=0$; using this, we obtain the following quadratic equation for the profit share:

$$
s(1-\delta) m^{2}-\Theta m+\varepsilon(\Gamma-n)=0, \quad \text { where } \Theta \equiv s\left(\gamma-\varepsilon u_{n}\right)+s(\Gamma-n)+\varepsilon>0 .
$$

From equation (21), we obtain two real and distinct roots. However, the larger root corresponds to the medium-run equilibrium value. ${ }^{8}$

$$
m^{*}=\frac{\Theta+\sqrt{\Theta^{2}-4 s \varepsilon(1-\delta)(\Gamma-n)}}{2 s(1-\delta)} .
$$

Using equation (22), we obtain the rates of capacity utilization and employment in the medium-run equilibrium:

$$
\begin{aligned}
& u^{*}=\frac{\delta m^{*}+\left(\gamma-\varepsilon u_{n}\right)}{s m^{*}-\varepsilon}, \\
& e^{*}=\left(\frac{m^{*}-\Gamma}{\lambda}\right)^{\frac{1}{\beta}}
\end{aligned}
$$

For the rate of capacity utilization to be positive, we need $s m^{*}-\varepsilon>0$, which can be rewritten as $m^{*}>\varepsilon / s$. In addition, we assume that $m^{*}, u^{*}$, and $e^{*}$ are larger than zero and less than unity.

By examining the system of differential equations (18), (19), and (20), we obtain the following proposition with regard to the stability of the medium-run equilibrium.

Proposition 2. Suppose that the coefficient of the investment function with respect to the rate of capacity utilization is not extremely large. Then, the medium-run equilibrium is locally stable.

Proof. See Appendix D.

In the following analysis, we assume that the coefficient of the investment function is not extremely large.

\subsection{Medium-run comparative statics analysis}

\subsubsection{Kalecki type investment function}

Table 1 summarizes the results of the comparative statics analysis in the medium-run equilibrium with the Kalecki investment function. ${ }^{9}$ These results are the same as those obtained

\footnotetext{
${ }^{8}$ See Appendix B.

${ }^{9} \mathrm{We}$ omit the partial derivatives because of spatial limitations.
} 
from the conventional Kaleckian model except for the results of the rate of employment that is not analyzed in the conventional models. For example, a rise in the capitalists' savings rate lowers the rate of capital accumulation, which is also known as the paradox of thrift. In our model, the profit share is an endogenous variable, and thus, we cannot directly relate a rise in $m$ to the changes in $u$ and $g$. Accordingly, we relate a rise in $m_{f}\left(m_{w}\right)$ to the changes in $u$ and $g$. A rise in $m_{f}\left(m_{w}\right)$ increases the profit share and lowers the rate of utilization and the rate of capital accumulation. In this sense, the medium-run equilibrium corresponds to both the stagnationist regime and the wage-led growth regime.

Table 1: Medium-run comparative statics analysis-Kalecki type investment function

\begin{tabular}{cccc}
\hline \hline & $s$ & $n$ & $m_{f}, m_{w}, \theta$ \\
\hline$u^{*}$ & - & + & - \\
$m^{*}$ & - & - & + \\
$e^{*}$ & - & - & - \\
$g^{*}$ & - & + & - \\
\hline \hline
\end{tabular}

We now look at the effect of a rise in $\theta$ on the rate of employment. In the Kalecki type investment function, an increase in the bargaining power of the firms decreases the rate of employment in the medium-run equilibrium. In other words, an increase in the bargaining power of the workers decreases the rate of unemployment. This result contrasts sharply with the neoclassical assertion that lowering the bargaining power of the labor union decreases the rate of unemployment.

\subsubsection{MB type investment function}

Table 2 summarizes the results of the comparative statics analysis in the medium-run equilibrium with the MB type investment function. ${ }^{10}$ The columns that contain two opposing signs indicate that two results are obtained depending on the conditions.

Using the MB type investment function, we obtain results that are different from the results in the Kalecki type investment function.

As in the case with the Kalecki type investment function, we define the regime using $m_{f}$ and $m_{w}$. First, a rise in $m_{f}\left(m_{w}\right)$ decreases the rate of capacity utilization, which means that the medium-run equilibrium is the stagnationist regime. ${ }^{11}$ Second, a rise in $m_{f}\left(m_{w}\right)$ either

\footnotetext{
${ }^{10} \mathrm{We}$ omit the partial derivatives because of spatial limitations.

${ }^{11}$ When using a linear MB type investment function like in the present paper, we cannot obtain the exhilarationist regime. For details, see Blecker (2002).
} 
Table 2: Medium-run comparative statics analysis-MB type investment function

\begin{tabular}{cccc}
\hline \hline & $s$ & $n$ & $m_{f}, m_{w}, \theta$ \\
\hline$u^{*}$ & - & + & - \\
$m^{*}$ & - & - & + \\
$e^{*}$ & - & - & $-/+$ \\
$g^{*}$ & - & $+/-$ & $-/+$ \\
\hline \hline
\end{tabular}

decreases (the wage-led growth regime) or increases (the profit-led growth regime) the rate of capital accumulation.

When the medium-run equilibrium is the profit-led growth regime, a rise in the bargaining power of the firms increases the rate of employment. In contrast, when the medium-run equilibrium is the wage-led growth, a rise in the bargaining power of the firms decreases the rate of employment. Therefore, the relationship between the bargaining power and the rate of employment differs between the two regimes. ${ }^{12}$

\section{Long-run equilibrium}

In the long run, the normal rate of capacity utilization and the expected rate of growth are adjusted. We assume that in the long run, the medium-run equilibrium is always attained: the medium-run equilibrium values $u^{*}, m^{*}$, and $e^{*}$ correspond to the actual values in the long run. Following Dutt (1997), Lavoie et al. (2004), and Cassetti (2006), we describe the long-run dynamics. ${ }^{13}$

First, we assume that the normal rate of capacity utilization is adjusted according to the gap between the actual rate of capacity utilization and the normal rate of capacity utiliza-

\footnotetext{
${ }^{12}$ Similar results are also obtained in Sasaki (2010).

${ }^{13}$ In the long run as considered in Cassetti (2006), in addition to the normal rate of capacity utilization and the expected rate of growth, the normal rate of profit and the drop-out ratio of capital equipment are also adjusted: the four variables are adjusted in the long run. In the present paper, we consider only the adjustments of the normal rate of capacity utilization and the expected rate of growth because we think that these two variables are essential in the long run.
} 
tion. $^{14}$

$$
\dot{u}_{n}=\phi\left(u^{*}-u_{n}\right), \quad \phi>0,
$$

where $\phi$ denotes the speed of adjustment. Note that we have $u^{*}=u^{*}\left(u_{n}, \gamma\right)$ from the mediumrun analysis.

Second, we assume that the expected rate of growth is adjusted according to the gap between the actual growth rate and the expected rate of growth:

$$
\dot{\gamma}=\eta\left(g^{*}-g_{d}^{*}\right), \quad \eta>0,
$$

where $\eta$ denotes the speed of adjustment. Note that when $u=u_{n}$, we have $g_{d}^{*}=\gamma+\delta r^{*}$ for the Kalecki type investment function and $g_{d}^{*}=\gamma+\delta m^{*}$ for the MB type investment function. Using this, for both the Kalecki type and the MB type investment functions, equation (26) can be rewritten as follows:

$$
\dot{\gamma}=\eta \varepsilon\left(u^{*}-u_{n}\right)
$$

\subsection{Kalecki type investment function}

The system of differential equations composed of equations (25) and (27) takes a special form called a zero-root system. ${ }^{15}$ In the zero-root system, one eigenvalue of the Jacobian matrix is zero. ${ }^{16}$

The long-run equilibrium is a situation wherein $\dot{u}_{n}=\dot{\gamma}=0$. From equations (25) and (27), we obtain $u^{*}\left(u_{n}, \gamma\right)=u_{n}$, which yields the following relationship between $u_{n}$ and $\gamma:{ }^{17}$

$$
\gamma=\frac{(s-\delta)(\Gamma-n) u_{n}}{1-s u_{n}}
$$

\footnotetext{
${ }^{14}$ The empirical validity of the simultaneous adjustments of the normal rate of capacity utilization and the expected rate of growth is supported by Lavoie et al. (2004). In contrast, Skott (2010) criticizes Lavoie et al. (2004): their estimated empirical hysteresis equation bears no relation to the theoretical model. For debates with regard to the long-run adjustment of the normal rate of capacity utilization, see also Hein, Lavoie, and van Treeck (2010a, b) and Skott and Zipperer (2010).

${ }^{15}$ For solutions to the zero-root system, see Giavazzi and Wyplosz (1985).

${ }^{16}$ The other eigenvalue is the trace of the Jacobian matrix.

${ }^{17}$ Using $u^{*}\left(u_{n}, \gamma\right)=u_{n}$ and (16), we obtain

$$
\frac{\gamma-\varepsilon u_{n}}{(s-\delta) m^{*}\left(u_{n}, \gamma\right)-\varepsilon}=u_{n} .
$$

Because $m^{* *}=[s \gamma+(s-\delta)(\Gamma-n)] /(s-\delta)$ in the long-run equilibrium, by replacing $m^{*}\left(u_{n}, \gamma\right)$ with $m^{* *}=$ $[s \gamma+(s-\delta)(\Gamma-n)] /(s-\delta)$ and rearranging the resultant expression, we obtain (28).
} 
On the $u_{n}-\gamma$ plane, this is an upward-sloping curve through the origin with $u_{n}=1 / \mathrm{s}$ being an asymptote. The points on this curve correspond to the long-run equilibria. However, not all the points on this curve are long-run equilibria and some constraints are imposed. ${ }^{18}$

From the two adjustment equations (25) and (27), we obtain the relationship $\dot{\gamma}=(\eta \varepsilon / \phi) \dot{u}_{n}$, from which we have the constant ratio $\gamma=(\eta \varepsilon / \phi) u_{n}$ along the transitional process. Hence, with initial conditions $\gamma(0)$ and $u_{n}(0)$, the transitional process is given by the following upward-sloping straight line:

$$
\gamma(t)=\frac{\eta \varepsilon}{\phi} u_{n}(t)+\gamma(0)-\frac{\eta \varepsilon}{\phi} u_{n}(0)
$$

The intersection of equations (28) and (29) yields the long-run equilibrium. It follows from this that the long-run equilibrium depends on the initial conditions $\gamma(0)$ and $u_{n}(0)$ : different initial conditions produce different long-run equilibria. Therefore, in this sense, the long-run equilibrium shows path-dependency.

Figure 1 shows the long-run phase diagram. First, we consider an economy that starts from point $A$. In this case, the long-run equilibrium is given by point $E_{1}$. Next, we consider an economy that starts from point $\mathrm{B}$. In this case, the long-run equilibrium is given by point $\mathrm{E}_{2}$

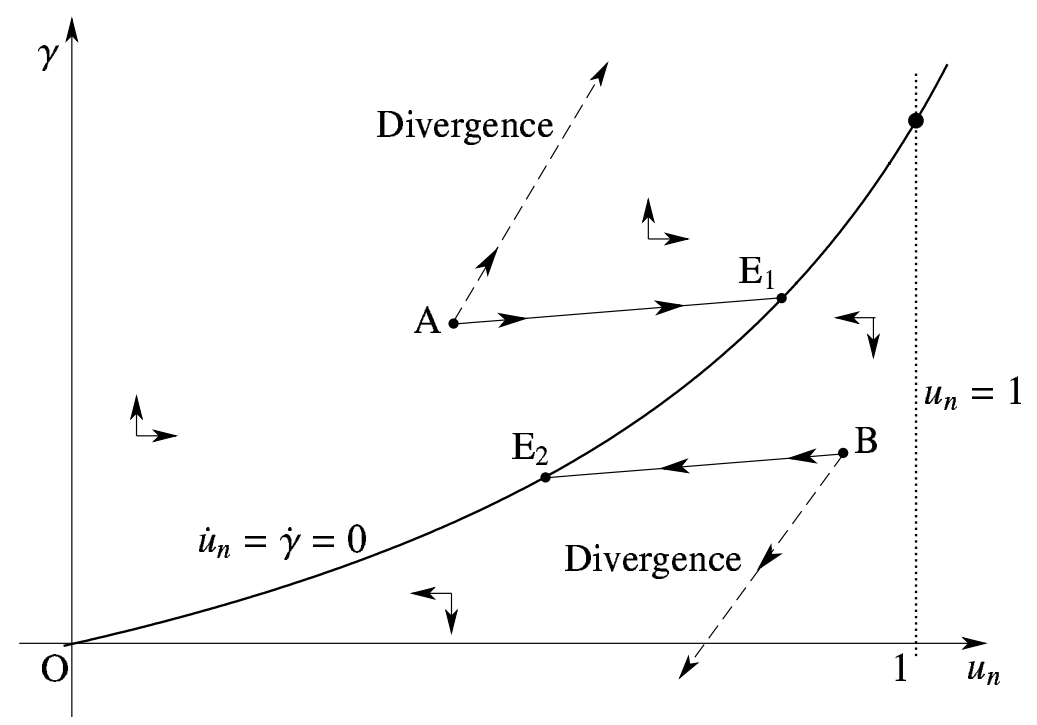

Figure 1: Long-run phase diagram

\footnotetext{
${ }^{18}$ Strictly speaking, we need additional conditions: $u^{*}, m^{*}$, and $e^{*}$ should be more than zero and less than unity in the medium run, $u_{n}$ should be more than zero and less than unity in the long run, etc. In the following analysis, we omit those conditions for the sake of simplicity.
} 
When $\gamma^{* *}$ is determined, the other long-run equilibrium values are determined as follows:

$$
\begin{aligned}
g^{* *} & =\frac{s \gamma^{* *}}{s-\delta}, \\
g_{a}^{* *} & =\frac{s \gamma^{* *}-n(s-\delta)}{s-\delta}, \\
m^{* *} & =\frac{s \gamma^{* *}+(s-\delta)(\Gamma-n)}{s-\delta}, \\
u^{* *} & =\frac{\gamma^{* *}}{s \gamma^{* *}+(s-\delta)(\Gamma-n)}, \\
e^{* *} & =\left[\frac{s \gamma^{* *}-n(s-\delta)}{\lambda(s-\delta)}\right]^{\frac{1}{\beta}} .
\end{aligned}
$$

The long-run equilibrium values are denoted with “**." Note that in addition to $u_{n}(0)$ and $\gamma(0), \gamma^{* *}$ depends on the initial conditions.

The long-run equilibrium is not always stable. If the coefficient $\eta \varepsilon / \phi$, the slope of the path, is extremely large, then the economy cannot reach the long-run equilibrium. In addition, even if the size of $\eta \varepsilon / \phi$ is modest, it is possible that the economy does not reach the long-run equilibrium depending on the initial condition. Let us return to figure 1 . The solution path denoted by the broken line starting from point A crosses the locus $\dot{u}_{n}=\dot{\gamma}=0$ at a point where $u_{n}$ exceeds unity, and consequently, the path is divergent. Moreover, the solution path denoted by the broken line starting from point B does not cross the locus $\dot{u}_{n}=\dot{\gamma}=0$. Therefore, both the size of adjustment and the initial condition are crucial for stable convergence to the long-run equilibrium.

We conduct a comparative statics analysis in the long-run equilibrium by assuming that the equilibrium is stable. Here, we investigate $u_{n}$ and $\gamma$. First, when $s$ or $\Gamma$ increases, the locus $\dot{u}_{n}=\dot{\gamma}=0$ rotates counterclockwise around the origin. Because the solution path is unaffected by the change in $s$ or $\Gamma$, the intersection of the solution path and the locus move toward the lower left. Therefore, both $u_{n}^{* *}$ and $\gamma^{* *}$ decrease. Second, when $n$ increases, the locus rotates clockwise. Because the solution path is unaffected by the change in $n$, both $u_{n}^{* *}$ and $\gamma^{* *}$ increase. These results are summarized in table 3.

Table 3: Long-run comparative statics analysis-Kalecki type investment function

\begin{tabular}{lccc}
\hline \hline & $s$ & $n$ & $m_{f}, m_{w}, \theta$ \\
\hline$\gamma^{* *}$ & - & + & - \\
\hline$u^{* *}$ & - & + & - \\
\hline \hline
\end{tabular}

We investigate the regime obtained in the long-run equilibrium. As in the medium-run 
equilibrium, we define the regime using $m_{f}$ and $m_{w}$. Depending on whether or not the expected growth rate $\gamma$ is adjusted, we obtain different results. ${ }^{19}$

Differentiating $u^{* *}$ and $g^{* *}$ with respect to $\Gamma$, we obtain

$$
\begin{aligned}
\frac{d u^{* *}}{d \Gamma} & =\frac{(s-\delta)(\Gamma-n) \frac{d \gamma^{* *}}{d \Gamma}-\gamma(s-\delta)}{[s \gamma+(s-\delta)(\Gamma-n)]^{2}}<0, \\
\frac{d g^{* *}}{d \Gamma} & =\frac{s}{s-\delta} \frac{d \gamma^{* *}}{d \Gamma} \leq 0 .
\end{aligned}
$$

First, when the expected rate of growth is not adjusted, we have $d \gamma^{* *} / d \Gamma=0$, and consequently, a rise in $m_{f}$ decreases the rate of capacity utilization but does not affect the rate of capital accumulation. Therefore, the long-run equilibrium is the stagnationist regime and not the wage-led growth regime or the profit-led growth regime. These results are explained as follows. When $\gamma$ is not adjusted, we can regard $\gamma$ as an exogenous variable. $g^{* *}$ depends on $\gamma$ but $\gamma$ does not depend on $m_{f}$, which implies that $g^{* *}$ does not depend on $m_{f}$. Therefore, the long-run equilibrium is neither the wage-led growth regime nor the profit-led growth regime. On the other hand, $u^{* *}$ depends on $m_{f}$ through $\Gamma$. Therefore, even if $\gamma$ is an exogenous variable, a rise in $m_{f}$ decreases $u^{* *}$.

Second, when the expected rate of growth is adjusted, we have $d \gamma^{* *} / d \Gamma<0$, and consequently, a rise in $m_{f}$ decreases both the rate of capacity utilization and the rate of capital accumulation in the long-run equilibrium. Therefore, the long-run equilibrium is both the stagnationist regime and the wage-led growth regime. In other words, if the expected rate of growth is adjusted, even in the long run, we obtain properties that are typical of the Kaleckian model. The reasoning is explained as follows. Both $g^{* *}$ and $u^{* *}$ depend on $\gamma^{* *}$. When the expected rate of growth is adjusted, $\gamma$ becomes an endogenous variable, and hence, $\gamma^{* *}$ is decreasing in $m_{f}$. Therefore, a rise in $m_{f}$ leads to a decrease in $\gamma^{* *}$, which leads to a decrease in both $u^{* *}$ and $g^{* *}$.

We now focus on the effect of a rise in the capitalists' savings rate on the rate of capacity utilization and the rate of capital accumulation in the long-run equilibrium. Differentiating $u^{* *}$ and $g^{* *}$ with respect to $s$, we obtain the following equations:

$$
\begin{aligned}
\frac{d u^{* *}}{d s} & =\frac{(s-\delta)(\Gamma-n) \frac{d \gamma^{* *}}{d s}-\gamma[\gamma+(\Gamma-n)]}{[s \gamma+(s-\delta)(\Gamma-n)]^{2}}<0, \\
\frac{d g^{* *}}{d s} & =\frac{s}{s-\delta} \frac{d \gamma^{* *}}{d s}-\frac{\delta \gamma^{* *}}{(s-\delta)^{2}}<0 .
\end{aligned}
$$

\footnotetext{
${ }^{19}$ When $\gamma$ is fixed and only $u_{n}$ is adjusted in our model, the resultant long-run equilibrium is always stable. On the other hand, when $u_{n}$ is fixed and only $\gamma$ is adjusted, the resultant long-run equilibrium is necessarily unstable. Therefore, we do not examine this latter case.
} 
While $u^{* *}$ and $g^{* *}$ depend directly on $s$, these values depend indirectly on $s$ through $\gamma^{* *}$. From this, we can see that both $u^{* *}$ and $g^{* *}$ are affected by a change in the savings rate irrespective of whether the expected rate of growth is adjusted or not. From equations (37) and (38), we find that a rise in $s$ decreases both $u^{* *}$ and $g^{* *}$. That is, a rise in the capitalists' savings rate lowers both the rate of capacity utilization and the rate of capital accumulation in the long-run equilibrium. ${ }^{20}$ Therefore, even in the long run, the paradox of thrift still holds, which is a characteristic of the Kaleckian growth model.

In turn, we investigate the long-run relationship between the rate of employment and the bargaining power. Since, in the long-run equilibrium, the relation $g_{a}^{* *}=g^{* *}-n$ holds and $g_{a}$ is increasing in $e$, the effect of $\Gamma$ (i.e., the effect of $\theta$ ) on $e^{* *}$ is the same as the effect of $\Gamma$ on $g^{* *}$. First, when the expected rate of growth is not adjusted, the bargaining power of the firms is unrelated to the rate of employment because $\theta$ does not appear in equation (34). Second, when the expected rate of growth is adjusted, a rise in the bargaining power of the firms lowers the rate of employment in the long-run equilibrium, which is the same result as that in the medium-run equilibrium.

\subsection{MB type investment function}

In the long-run equilibrium, both $u_{n}$ and $\gamma$ satisfy the following relationship: ${ }^{21}$

$$
\gamma=\frac{(\Gamma-n)\left(s u_{n}-\delta\right)}{1-s u_{n}}
$$

As in the Kalecki type investment function, this is an upward-sloping curve.

The long-run solution path satisfies the following relationship:

$$
\gamma(t)=\frac{\eta \varepsilon}{\phi} u_{n}(t)+\gamma(0)-\frac{\eta \varepsilon}{\phi} u_{n}(0) .
$$

This is identical to the solution path in the Kalecki type investment function. The intersection of (39) and (40) determines the long-run equilibrium.

\footnotetext{
${ }^{20}$ We must pay attention to the case where the expected rate of growth is not adjusted. Note that in the present paper, both the rate of profit and the rate of capacity utilization are explanatory variables of the investment function. In contrast, if the investment function includes only the rate of capacity utilization as the explanatory variable, that is, if $\delta=0$, we have $g^{* *}=\gamma^{* *}$. If the expected rate of growth is not adjusted, that is, if $\gamma$ is fixed, then $g^{* *}$ is independent of $s$, and consequently, the paradox of thrift is not obtained. Therefore, the results in the present paper depend on the specification of the investment function.

${ }^{21}$ Equation (39) can be obtained by the same procedure as that explained in footnote 17.
} 
When $\gamma^{* *}$ is determined, the other long-run equilibrium values are determined as follows:

$$
\begin{aligned}
g^{* *} & =\frac{\gamma^{* *}+\delta(\Gamma-n)}{1-\delta}, \\
g_{a}^{* *} & =\frac{\gamma^{* *}-n+\delta \Gamma}{1-\delta}, \\
m^{* *} & =\frac{\gamma^{* *}+\Gamma-n}{1-\delta}, \\
u^{* *} & =\frac{\gamma^{* *}+\delta(\Gamma-n)}{s\left[\gamma^{* *}+(\Gamma-n)\right]}, \\
e^{* *} & =\left[\frac{\gamma^{* *}-n+\delta \Gamma}{\lambda(1-\delta)}\right]^{\frac{1}{\beta}} .
\end{aligned}
$$

The long-run phase diagram is almost the same as that of the Kalecki type investment function, and thus, we omit it. Therefore, the long-run equilibrium is stable in some cases and unstable in others. Table 4 shows the results of the long-run comparative statics analysis given the stability of the long-run equilibrium. The reasons why we obtain such results are the same as those in the Kalecki type investment function case. A rise in $s$ or $\Gamma$ rotates the locus of $\dot{u}_{n}=\dot{\gamma}=0$ counterclockwise, and a rise in $n$ rotates the locus clockwise. In either case, the solution path does not shift. Therefore, we obtain the results shown in table 4.

Table 4: Long-run comparative statics analysis-MB type investment function

\begin{tabular}{lccc}
\hline \hline & $s$ & $n$ & $m_{f}, m_{w}, \theta$ \\
\hline$\gamma^{* *}$ & - & + & - \\
\hline$u^{* *}$ & - & + & - \\
\hline
\end{tabular}

Let us investigate the long-run equilibrium regime. Differentiating $u^{* *}$ and $g^{* *}$ with respect to $\Gamma$, we obtain

$$
\begin{aligned}
\frac{d u^{* *}}{d \Gamma} & =\frac{s(1-\delta)(\Gamma-n) \frac{d \gamma^{* *}}{d \Gamma}-s \gamma(1-\delta)}{[s \gamma+s(\Gamma-n)]^{2}}<0, \\
\frac{d g^{* *}}{d \Gamma} & =\frac{1}{1-\delta}\left(\frac{d \gamma^{* *}}{d \Gamma}+\delta\right) .
\end{aligned}
$$

First, we consider the case where the expected rate of growth is not adjusted. Since $\gamma$ is fixed and constant in this case, we obtain $d \gamma^{* *} / d \Gamma=0$. From this, we get that a rise in $m_{f}$ decreases $u^{* *}$ but increases $g^{* *}$. Therefore, the long-run equilibrium is the stagnationist regime and the profit-led growth regime. Recall that in the medium-run equilibrium of the MB type investment function, we obtain either the wage-led growth regime or the profit-led 
growth regime depending on the conditions. In contrast, we obtain only profit-led growth in the long-run equilibrium when the expected rate of growth is not adjusted.

Next, we consider the case where the expected rate of growth is adjusted. In this case, we obtain $d \gamma^{* *} / d \Gamma<0$. However, the sign of equation (47) is undetermined: the sign depends on the size of $\delta$ of the investment function (4). If $\delta$ is smaller than the absolute value of $d \gamma^{* *} / d \Gamma$, the long-run equilibrium is the wage-led growth regime. On the other hand, if $\delta$ is larger than the absolute value of $d \gamma^{* *} / d \Gamma$, then the long-run equilibrium is the profit-led growth regime. Note that when $\delta$ is small (large), the medium-run equilibrium tends to be the wage-led (profit-led) growth regime. This fact suggests that when the medium-run equilibrium is the wage-led (profit-led) growth regime, the long-run equilibrium is also the wage-led (profit-led) growth regime. ${ }^{22}$

We now focus on the effect of a rise in the capitalists' savings rate on the rate of capacity utilization and the rate of capital accumulation in the long-run equilibrium. Differentiating $u^{* *}$ and $g^{* *}$ with respect to $s$, we obtain the following equations:

$$
\begin{aligned}
\frac{d u^{* *}}{d s} & =\frac{s(1-\delta)(\Gamma-n) \frac{d \gamma^{* *}}{d s}-[\gamma+\delta(\Gamma-n)][\gamma+(\Gamma-n)]}{[s \gamma+s(\Gamma-n)]^{2}}<0, \\
\frac{d g^{* *}}{d s} & =\frac{1}{1-\delta} \frac{d \gamma^{* *}}{d s} \leq 0 .
\end{aligned}
$$

First, when $\gamma$ is not adjusted, a rise in the savings rate lowers the rate of capacity utilization. However, the rate of capital accumulation in the long-run equilibrium is independent of $s$ when $d \gamma^{* *} / d s=0$, and consequently, the paradox of thrift does not hold. Second, when $\gamma$ is adjusted and accordingly, $d \gamma^{* *} / d s<0$, a rise in the savings rate increases both the rate of capacity utilization and the rate of capital accumulation. Therefore, the paradox of thrift holds. In the Kalecki type investment function case, we obtain the paradox of thrift irrespective of whether or not the expected rate of growth is adjusted. However, in the MB type investment function case, we obtain the paradox of thrift only when the expected rate of growth is adjusted.

We consider the long-run relationship between the rate of employment and the bargaining power. From the structure of the model, the effect of a rise in $m_{f}$ on the rate of employment is identical to the effect of a rise in $\theta$ on the rate of employment. As a result, when the long-run equilibrium is the wage-led (profit-led) growth regime, a rise in the bargaining power of the firms decreases (increases) the rate of employment. This result is the same as that obtained in the medium-run equilibrium.

\footnotetext{
${ }^{22} \mathrm{We}$ can show that such a relationship actually holds using numerical examples. For this, see Appendix E.
} 


\subsection{Summary}

Finally, we summarize the results obtained in the medium-run and long-run equilibria.

Table 5: Classification of the regimes in the medium-run and long-run equilibria

\begin{tabular}{rcccc}
\hline \hline & $\begin{array}{c}\text { Kalecki } \\
\text { (medium-run) }\end{array}$ & $\begin{array}{c}\text { MB } \\
\text { (medium-run) }\end{array}$ & $\begin{array}{c}\text { Kalecki } \\
\text { (long-run) } \\
\gamma \text { : fixed; adjusted }\end{array}$ & $\begin{array}{c}\text { MB fixed; adjusted } \\
\text { (long-run) }\end{array}$ \\
\hline Stagnationist & $\circ$ & $\circ$ & $\circ, \circ$ & $\circ, \circ$ \\
Wage-led growth & $\circ$ & $\circ$ & $\times, \circ$ & $\times, \circ$ \\
Profit-led growth & $\times$ & $\circ$ & $\times, \times$ & $\circ, \circ$ \\
\hline \hline
\end{tabular}

In table 5, the symbol "o" indicates that the corresponding regime is obtained whereas the symbol " $x$ " indicates that the corresponding regime is not obtained. For example, in the medium-run equilibrium with the Kalecki type investment function, both the stagnationist and the wage-led growth regimes are obtained whereas the profit-led growth regime is not obtained. In the columns headed with "long-run," the left cell corresponds to the case where the expected rate of growth is fixed while the right cell corresponds to the case where the expected rate of growth is adjusted. For example, in the long-run equilibrium with the MB type investment function, the wage-led growth regime is not obtained when $\gamma$ is fixed, while the wage-led growth regime is obtained when $\gamma$ is adjusted.

\section{Conclusions}

This paper presents a long-run Kaleckian model, and investigates which regime is obtained in the long-run equilibrium. For this purpose, we use the Kalecki type investment function and the MB type investment function. Moreover, we discuss the equilibrium rate of employment that is not considered in detail in the existing Kaleckian models. The results are summarized as follows.

When the expected rate of growth is not adjusted, the long-run equilibrium with the Kalecki type investment function is neither the wage-led growth regime nor the profit-led growth regime, while the long-run equilibrium with the MB investment function is the profitled growth regime.

When the expected rate of growth is adjusted, the long-run equilibrium with the Kalecki type investment function is the wage-led growth regime; on the other hand, the long-run equilibrium with the MB type investment function is the wage-led growth regime if the medium-run equilibrium is the wage-led growth regime, but is the profit-led growth regime 
if the medium-run equilibrium is the profit-led growth regime. Therefore, we find that the important characteristics of the Kaleckian model are also observed in the long run.

The relationship between the rate of employment and the bargaining power of the firms is as follows. In the medium-run equilibrium, a rise in the bargaining power of the firms decreases the rate of employment if the equilibrium is the wage-led growth regime, but increases the rate of employment if the equilibrium is the profit-led growth regime. In the long-run equilibrium with the Kalecki type investment function, the rate of employment is free from the bargaining power of the firms if the expected rate of growth is not adjusted.

From these observations, we find that the adjusting of the expected rate of growth is decisive in determining whether or not the characteristics of the medium-run equilibrium are reflected in the characteristics of the long-run equilibrium. Naturally, the adjustment of the normal rate of capacity utilization also affects the results. Therefore, in the future, we intend to focus on the empirical tests for the validity of these long-run adjustments. In addition, since the type of the investment function affects the results, the empirical tests for the investment function will also be looked into.

\section{References}

Amadeo, E. 1986. Notes on capacity utilisation, distribution and accumulation. Contributions to Political Economy 5, no. 1: 83-94.

Auerbach, P., and P. Skott. 1988. Concentration, competition and distribution-a critique of theories of monopoly capital. International Review of Applied Economics 2, no. 1: 42-61.

Bhaduri, A. 2006. Endogenous economic growth: a new approach. Cambridge Journal of Economics 30, no. 1: 69-83.

Bhaduri, A., and S. Marglin. 1990. Unemployment and the real wage: the economic basis for contesting political ideologies. Cambridge Journal of Economics 14, no. 4: 375-393.

Blecker, R.A. 2002. Distribution, demand and growth in neo-Kaleckian macro-models. In The Economics of Demand-led Growth, Challenging the Supply-side Vision of the Long Run, ed. M. Setterfield. Cheltenham: Edward Elgar.

Cassetti, M. 2002. Conflict, inflation, distribution and terms of trade in the Kaleckian model. In The Economics of Demand-led Growth, Challenging the Supply-side Vision of the Long Run, ed. M. Setterfield. Cheltenham: Edward Elgar. 
Cassetti, M. 2003. Bargaining power, effective demand and technical progress: a Kaleckian model of growth. Cambridge Journal of Economics 27, no. 3: 449-464.

Cassetti, M. 2006. A note on the long-run behaviour of Kaleckian models. Review of Political Economy 18, no. 4: 497-508.

Duménil, G., and D. Lévy. 1999. Being Keynesian in the short term and classical in the long term: the traverse to classical long-term equilibrium. The Manchester School 67, no. 6: 684-716.

Dutt, A.K. 1987. Alternative closures again: a comment on growth, distribution and inflation. Cambridge Journal of Economics 11, no. 1: 75-82.

Dutt, A.K. 1992. Conflict inflation, distribution, cyclical accumulation and crises. European Journal of Political Economy 8, no. 4: 579-597.

Dutt, A.K. 1997. Equilibrium, path dependence and hysteresis in post-Keynesian models. In Markets, Unemployment and Economic Policy: Essays in Honour of Geoff Harcourt, Vol. 2, eds. P. Arestis, G. Palma, and M. Sawyer. London: Routledge.

Dutt, A.K. 2006. Aggregate demand, aggregate supply and economic growth. International Review of Applied Economics 20, no. 3: 319-336.

Flaschel, P., and P. Skott. 2006. Steindlian models of growth and stagnation. Metroeconomica 57, no. 3: 303-338.

Giavazzi, F., and C. Wyplosz. 1985. The zero root problem: a note on the dynamic determination of the stationary equilibrium in linear models. Review of Economic Studies 52, no. 2: 353-357.

Hein, E., Lavoie, M., and T. van Treeck. 2010a. Some instability puzzles in Kaleckian models of growth and distribution: a critical survey. Cambridge Journal of Economics 14, no. 5: 375-393.

Hein, E., Lavoie, M., and T. van Treeck. 2010b. Harrodian instability and the 'normal rate' of capacity utilization in Kaleckian models of distribution and growth-a survey. Metroeconomica forthcoming.

Lavoie, M. 1992. Foundations of Post-Keynesian Economic Analysis. Cheltenham: Edward Elgar. 
Lavoie, M. 1995. The Kaleckian model of growth and distribution and its neo-Ricardian and neo-Marxian critiques. Cambridge Journal of Economics 19, no. 6: 789-818.

Lavoie, M. 2003. Kaleckian effective demand and Sraffian normal prices: towards a reconciliation. Review of Political Economy 15, no. 1: 53-74.

Lavoie, M. 2006. Introduction to Post-Keynesian Economics. New York: Palgrave Macmillan.

Lavoie, M., G. Rodríguez, and M. Seccareccia. 2004. Similitudes and discrepancies in postKeynesian and Marxist theories of investment: a theoretical and empirical investigation. International Review of Applied Economics 18, no. 2: 127-149.

Lima, G.T. 2004. Endogenous technological innovation, capital accumulation and distributional dynamics. Metroeconomica 55, no. 4: 386-408.

Marglin, S., and A. Bhaduri. 1990. Profit squeeze and Keynesian theory. In The Golden Age of Capitalism: Reinterpreting the Postwar Experience, eds. S. Marglin and J. Schor. Oxford: Clarendon Press.

Park, M.S. 1997. Normal values and average values. Metroeconomica 48, no. 2: 188-199.

Rowthorn, R.E. 1977. Conflict, inflation and money. Cambridge Journal of Economics 1, no. 3: 215-239.

Rowthorn, R.E. 1981. Demand, real wages and economic growth. Thames Papers in Political Economy, Autumn: 1-39.

Sasaki, H. 2010. Endogenous technological change, income distribution, and unemployment with inter-class conflict. Structural Change and Economic Dynamics 21, no. 2: 123 134.

Skott, P. 2010. Theoretical and empirical shortcomings of the Kaleckian investment function. Metroeconomica 59, no. 3: 441-478.

Skott, P., and B. Zipperer. 2010. An empirical evaluation of three post Keynesian models. Working Paper 2010-08, University of Massachusetts Amherst.

Stockhammer, E. 2004. Is there an equilibrium rate of unemployment in the long run? Review of Political Economy 16, no. 1: 59-77. 


\section{Appendix}

\section{A Medium-run equilibrium profit share: Kalecki type in- vestment function}

Let $m_{1}$ and $m_{2}$ be the roots of the quadratic equation (14). Calculating the discriminant of the equation, we find it to be positive. Hence, $m_{1}$ and $m_{2}$ are the two real distinct roots. Let $m_{1}<m_{2}$. We have

$$
\begin{aligned}
m_{1}+m_{2} & =\frac{\Theta}{s-\delta}>0, \\
m_{1} m_{2} & =\frac{\varepsilon(\Gamma-n)}{s-\delta}>0 .
\end{aligned}
$$

That is, both $m_{1}$ and $m_{2}$ are positive. The condition for the medium-run equilibrium value of the rate of capacity utilization to be positive is given by $(s-\delta) m-\varepsilon>0$. From this, we obtain $m>\varepsilon /(s-\delta)$, which is a constraint on $m^{*}$. Let $f(m)$ be the left-hand side of equation (14). Substituting $m=\varepsilon /(s-\delta)$ in $f(m)$, we obtain

$$
f(\varepsilon /(s-\delta))=-\frac{\varepsilon s\left(\gamma-\varepsilon u_{n}\right)}{s-\delta}<0 .
$$

This means that $m_{1}$ is smaller than $\varepsilon /(s-\delta)$ and that $m_{2}$ is larger than $\varepsilon /(s-\delta)$. Therefore, $m_{2}$ is the medium-run equilibrium value.

\section{B Medium-run equilibrium profit share: MB type invest- ment function}

Let $m_{1}$ and $m_{2}$ be the roots of the quadratic equation (21). Calculating the discriminant of the equation, we find it to be positive. Hence, $m_{1}$ and $m_{2}$ are the two real distinct roots. Let $m_{1}<m_{2}$. We have

$$
\begin{aligned}
m_{1}+m_{2} & =\frac{\Theta}{s(1-\delta)}>0, \\
m_{1} m_{2} & =\frac{\varepsilon(\Gamma-n)}{s(1-\delta)}>0 .
\end{aligned}
$$

That is, both $m_{1}$ and $m_{2}$ are positive. The condition for the medium-run equilibrium value of the rate of capacity utilization to be positive is given by $s m-\varepsilon>0$. From this, we obtain $m>\varepsilon / s$, which is a constraint on $m^{*}$. Let $f(m)$ be the left-hand side of equation (21). 
Substituting $m=\varepsilon / s$ in $f(m)$, we obtain

$$
f(\varepsilon / s)=-\frac{\varepsilon^{2}}{s}-\left(\gamma-\varepsilon u_{n}\right)-(1-\varepsilon)(\Gamma-n)<0 .
$$

This means that $m_{1}$ is smaller than $\varepsilon / s$ and that $m_{2}$ is larger than $\varepsilon / s$. Therefore, $m_{2}$ is the medium-run equilibrium value.

\section{Local stability of the medium-run equilibrium: Kalecki type investment function}

To investigate the local stability of the medium-run equilibrium, we linearize the system of differential equations (11), (12), and (13) around the equilibrium.

$$
\left(\begin{array}{c}
\dot{u} \\
\dot{m} \\
\dot{e}
\end{array}\right)=\left(\begin{array}{ccc}
J_{11} & J_{12} & 0 \\
0 & J_{22} & J_{23} \\
J_{31} & J_{32} & J_{33}
\end{array}\right)\left(\begin{array}{c}
u-u^{*} \\
m-m^{*} \\
e-e^{*}
\end{array}\right) .
$$

The elements of the Jacobian matrix $\mathbf{J}$ are given by

$$
\begin{aligned}
J_{11} & \equiv \frac{\partial \dot{u}}{\partial u}=-\alpha[(s-\delta) m-\varepsilon]<0, \\
J_{12} & \equiv \frac{\partial \dot{u}}{\partial m}=-\alpha(s-\delta) u<0, \\
J_{22} & \equiv \frac{\partial \dot{m}}{\partial m}=-(1-m)<0 \\
J_{23} & \equiv \frac{\partial \dot{m}}{\partial e}=(1-m) g_{a}^{\prime}(e)>0, \\
J_{31} & \equiv \frac{\partial \dot{e}}{\partial u}=-\frac{\alpha e[(s-\delta) m-\varepsilon]}{u}+e(\delta m+\varepsilon), \\
J_{32} & \equiv \frac{\partial \dot{e}}{\partial m}=-e[\alpha(s-\delta)-\delta u], \\
J_{33} & \equiv \frac{\partial \dot{e}}{\partial e}=-e g_{a}^{\prime}(e)<0 .
\end{aligned}
$$

All elements are evaluated at the equilibrium value; we omit “*” to avoid troublesome notations.

The characteristic equation of $\mathbf{J}$ is given by

$$
q^{3}+a_{1} q^{2}+a_{2} q+a_{3}=0
$$

where $q$ denotes a characteristic root. Each coefficient of equation $(\mathrm{C}-15)$ is given by

$$
\begin{aligned}
& a_{1}=-\operatorname{tr} \mathbf{J}=-\left(J_{11}+J_{22}+J_{33}\right), \\
& a_{2}=\left|\begin{array}{ll}
J_{22} & J_{23} \\
J_{32} & J_{33}
\end{array}\right|+\left|\begin{array}{cc}
J_{11} & 0 \\
J_{31} & J_{33}
\end{array}\right|+\left|\begin{array}{cc}
J_{11} & J_{12} \\
0 & J_{22}
\end{array}\right|=J_{22} J_{33}-J_{23} J_{32}+J_{11}\left(J_{33}+J_{22}\right),
\end{aligned}
$$




$$
a_{3}=-\operatorname{det} \mathbf{J}=-J_{11}\left(J_{22} J_{33}-J_{23} J_{32}\right)-J_{31} J_{12} J_{23},
$$

where $-a_{1}=\operatorname{tr} \mathbf{J}$ denotes the trace of $\mathbf{J} ; a_{2}$, the sum of the principal minors' determinants; and $-a_{3}=\operatorname{det} \mathbf{J}$, the determinant of $\mathbf{J}$.

The necessary and sufficient conditions for the local stability are $a_{1}>0, a_{2}>0, a_{3}>0$, and $a_{1} a_{2}-a_{3}>0$. Accordingly, we need to examine these inequalities.

$$
\begin{aligned}
& a_{1}=\underbrace{[(s-\delta) m-\varepsilon]}_{\equiv A>0} \alpha+\underbrace{(1-m)+e g_{a}^{\prime}(e)}_{\equiv B>0}=\underset{+}{A \alpha} \alpha+\underset{+}{B}>0, \\
& a_{2}=\underbrace{\left\{[(s-\delta) m-\varepsilon]\left[(1-m)+e g_{a}^{\prime}(e)\right]+(s-\delta)(1-m) e g_{a}^{\prime}(e)\right\}}_{\equiv C>0} \alpha+\underbrace{(1-\delta u)(1-m) e g_{a}^{\prime}(e)}_{\equiv D>0} \\
& =\underset{+}{C} \alpha+\underset{+}{D}>0 \\
& a_{3}=\underbrace{(1-m) e g_{a}^{\prime}(e)\{[(s-\delta) m-\varepsilon]+\varepsilon s u\}}_{\equiv E>0} \alpha=\underset{+}{E} \alpha>0, \\
& a_{1} a_{2}-a_{3}=\underbrace{A C}_{+} \alpha^{2}+(A D+B C-E) \alpha+\underbrace{B D}_{+} .
\end{aligned}
$$

Here, we have

$$
\begin{aligned}
A D+B C-E= & {[\underbrace{}_{+}(s-\delta) m-\varepsilon]\left[(1-m)+e g_{a}^{\prime}(e)\right]^{2} } \\
& +\underbrace{(s-\delta)(1-m) e g_{a}^{\prime}(e)}_{+}[e g_{a}^{\prime}(e)+\underbrace{1-m-\delta u m-\varepsilon u}_{\equiv \Lambda}] .
\end{aligned}
$$

$\Lambda$ will be positive if $\delta$ and $\varepsilon$ are not extremely large. When $\Lambda>0$, we obtain $A D+B C-E>0$, which leads to $a_{1} a_{2}-a_{3}>0$. Therefore, if $\Lambda>0$, the necessary and sufficient conditions for the local stability are satisfied. Note that $\Lambda>0$ is a sufficient condition for $a_{1} a_{2}-a_{3}>0$.

\section{Local stability of the medium-run equilibrium: MB type investment function}

The elements of the Jacobian matrix $\mathbf{J}$ that consists of equations (18), (19), and (20) are given by

$$
\begin{aligned}
& J_{11} \equiv \frac{\partial \dot{u}}{\partial u}=-\alpha(s m-\varepsilon)<0, \\
& J_{12} \equiv \frac{\partial \dot{u}}{\partial m}=-\alpha(s u-\delta), \\
& J_{22} \equiv \frac{\partial \dot{m}}{\partial m}=-(1-m)<0,
\end{aligned}
$$




$$
\begin{aligned}
& J_{23} \equiv \frac{\partial \dot{m}}{\partial e}=(1-m) g_{a}^{\prime}(e)>0, \\
& J_{31} \equiv \frac{\partial \dot{e}}{\partial u}=-\frac{\alpha e(s m-\varepsilon)}{u}+\varepsilon e, \\
& J_{32} \equiv \frac{\partial \dot{e}}{\partial m}=-\frac{e[\alpha(s u-\delta)-\delta u]}{u}, \\
& J_{33} \equiv \frac{\partial \dot{e}}{\partial e}=-e g_{a}^{\prime}(e)<0 .
\end{aligned}
$$

All elements are evaluated at the equilibrium value.

The coefficients of the characteristic equation are given by

$$
\begin{aligned}
& a_{1}=\underbrace{(s m-\varepsilon)}_{\equiv A>0} \alpha+\underbrace{(1-m)+e g_{a}^{\prime}(e)}_{\equiv B>0}=\underset{+}{A} \alpha+\underset{+}{B}>0, \\
& a_{2}=\underbrace{\left\{\frac{(1-m) e g_{a}^{\prime}(e)(s u-\delta)}{u}+(s m-\varepsilon)\left[(1-m)+e g_{a}^{\prime}(e)\right]\right\}}_{\equiv C>0} \alpha+\underbrace{(1-\delta)(1-m) e g_{a}^{\prime}(e)}_{\equiv D>0} \\
& =\underset{+}{C} \alpha+\underset{+}{D}>0, \\
& a_{3}=\underbrace{(1-m) e g_{a}^{\prime}(e)[(1-\delta)(s m-\varepsilon)+\varepsilon(s u-\delta)]}_{\equiv E>0} \alpha=\underset{+}{E} \alpha>0, \\
& a_{1} a_{2}-a_{3}=\underbrace{A C}_{+} \alpha^{2}+(A D+B C-E) \alpha+\underbrace{B D}_{+} .
\end{aligned}
$$

Here, we have

$$
\begin{aligned}
A D+B C-E= & \underbrace{(s m-\varepsilon)\left[(1-m)+e g_{a}^{\prime}(e)\right]^{2}}_{+} \\
& +\underbrace{(1-m) e g_{a}^{\prime}(e)(s u-\delta)}_{+}\left[\frac{e g_{a}^{\prime}(e)+\overbrace{1-m-\varepsilon u}^{\equiv \Lambda}}{u}\right] .
\end{aligned}
$$

$\Lambda$ is positive if $\varepsilon$ is not extremely large. When $\Lambda>0$, we obtain $A D+B C-E>0$, which leads to $a_{1} a_{2}-a_{3}>0$. Therefore, if $\Lambda>0$, the necessary and sufficient conditions for the local stability are satisfied. Note that $\Lambda>0$ is a sufficient condition for $a_{1} a_{2}-a_{3}>0$. 


\section{E Numerical examples in the long run: MB type invest- ment function}

Table 6 shows the parameters used for the numerical example. The initial values are set to $u_{n}(0)=0.5$ and $\gamma(0)=0.15$ (common to all cases). ${ }^{23}$ In these examples, only the sizes of the coefficients $\varepsilon$ and $\delta$ are different. The coefficient $\varepsilon$ is larger (smaller) than the coefficient $\delta$ in the wage-led (profit-led) growth regime example.

Table 6: Parameters used in the numerical examples

\begin{tabular}{ccccccccccccc}
\hline \hline & $\theta$ & $m_{f}$ & $m_{w}$ & $s$ & $n$ & $\varepsilon$ & $\delta$ & $\lambda$ & $\psi$ & $\alpha$ & $\phi$ & $\eta$ \\
\hline MB wage-led growth & 0.3 & 0.3 & 0.2 & 0.75 & 0.05 & 0.08 & 0.05 & 0.15 & 1 & 1 & 1 & 1 \\
MB profit-led growth & 0.3 & 0.3 & 0.2 & 0.75 & 0.05 & 0.04 & 0.09 & 0.15 & 1 & 1 & 1 & 1 \\
\hline \hline
\end{tabular}

Table 7 shows the results when the medium-run equilibrium is the wage-led growth regime. We increase the target profit share of the firms $m_{f}$ from 0.3 to 0.31 . From this, we have that a rise in $m_{f}$ lowers both the rate of capacity utilization and the rate of capital accumulation in the long-run equilibrium. In other words, in this numerical example, the long-run equilibrium is the stagnationist regime and the wage-led growth regime. Therefore, the long-run equilibrium regime is the same as the medium-run equilibrium regime.

Table 7: Example where the long-run equilibrium is the wage-led growth regime (the medium-run equilibrium is the wage-led growth regime)

\begin{tabular}{lccccccc}
\hline \hline & $u$ & $g$ & $m$ & $e$ & $u_{n}$ & $\gamma$ & $g_{a}$ \\
\hline Benchmark & 0.670 & 0.182 & 0.362 & 0.878 & 0.670 & 0.164 & 0.132 \\
$m_{f}: 0.3 \rightarrow 0.31$ & 0.664 & 0.181 & 0.364 & 0.875 & 0.664 & 0.163 & 0.131 \\
\hline \hline
\end{tabular}

Table 8 shows the results when the medium-run equilibrium is the profit-led growth regime. From this, we have that a rise in $m_{f}$ decreases the rate of capacity utilization and the rate of capital accumulation in the long-run equilibrium. In other words, in this numerical example, the long-run equilibrium is both the stagnationist regime and the profit-led growth regime. Therefore, the long-run equilibrium regime is the same as the medium-run equilibrium regime.

\footnotetext{
${ }^{23}$ Since the long-run equilibrium has a path-dependent property, different initial conditions produce different long-run equilibrium values.
} 
Table 8: Example where the long-run equilibrium is the profit-led growth regime (the medium-run equilibrium is the profit-led growth regime)

\begin{tabular}{lccccccc}
\hline \hline & $u$ & $g$ & $m$ & $e$ & $u_{n}$ & $\gamma$ & $g_{a}$ \\
\hline Benchmark & 0.6861 & 0.1908 & 0.3708 & 0.9388 & 0.6861 & 0.1574 & 0.1408 \\
$m_{f}: 0.3 \rightarrow 0.31$ & 0.6807 & 0.1909 & 0.3739 & 0.9392 & 0.6807 & 0.1572 & 0.1409 \\
\hline \hline
\end{tabular}

